\title{
Fragmentation and Correlation of Hadrons in ep Collisions
}

\author{
Artur Ukleja (on behalf of H1 and ZEUS Collaborations) \\ Warsaw University, Faculty of Physics, 00681 Warszawa, Hoza 69, \\ Poland and Deutsches Elektronen-Synchrotron 22607 Hamburg, Notkestr. 85, Germany
}

Received on 31 October, 2006; Modified on 14 November, 2006; Third version on 11 April, 2007;

Urth version on 12 April, 2007; Fifth version on 17 April, 2007

\begin{abstract}
Electron-proton $(e p)$ collisions at HERA can be regarded as an interaction between a virtual photon and the proton. The interaction gives a unique opportunity for studying particle production in the hadronic final state of the $e p$ collisions. This contribution reviews the momentum distribution of fragmented hadrons and strange meson and baryon production. Also the Bose-Einstein correlation length and azimuthal asymmetry were measured.
\end{abstract}

Keywords: Bose-Einstein correlation; Deep inelastic scattering; Parton fragmentation

\section{INTRODUCTION}

The description of the hadronic final state in deep inelastic scattering (DIS) is influenced by perturbative Quantum Chromodynamics (pQCD) in several ways, which can be calculated through exact matrix elements or leading-logarithm parton showers.

The process of parton fragmentation and hadronization can be studied in the measurements of the particle momentum distribution, particle production and Bose-Einstein correlations. The matrix element can be studied in the measurements of the particle momentum distribution and the distribution of the azimuthal angle.

\section{MOMENTUM DISTRIBUTION}

The normalised scaled-momentum distributions of charged particles, $x_{p}$, is shown in Fig. 1 as a function of the initial parton energy $Q$ for nine different intervals of $x_{p}$. This analysis has significantly higher statistics than previous studies [1-5], extending the kinematic range to higher $Q^{2}$ and the full range of $x_{p}$.

Moving from low to high $Q$ the spectrum becomes softer, i.e. there is a dramatic increase in the number of hadrons with a small share of the initial parton's momentum and a decrease in those hadrons with a large share. The observed dependence with $Q^{2}$ shows similar behavior as the scaling violation of the structure function, indicating that the observed dependence is a manifestation of the parton splitting. There is good agreement between $e p$ and $e^{+} e^{-}$, which provides a good demonstration of quark fragmentation universality.

\section{PARTICLE PRODUCTION}

Production of $K_{S}^{0}, \Lambda$ and $\bar{\Lambda}$ has been used to test QCD and to build phenomenological models extending QCD predictions beyond what can be calculated from first principles. Although, to a good approximation, the yield of hadrons in events with hadronic jets of high transverse energy does not depend on the type of colliding beam particles, this statement is not true when hadronic jets have low transverse energy. In
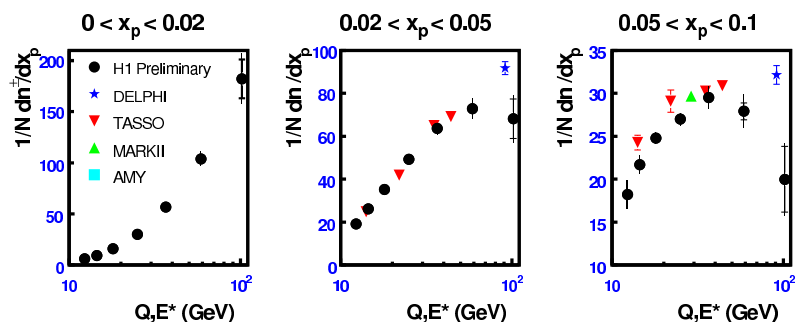

$0.1<\mathrm{x}_{\mathrm{p}}<0.2$

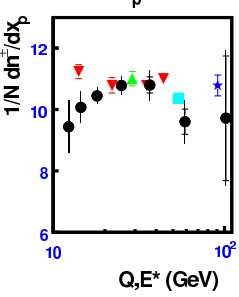

$0.2<x_{p}<0.3$

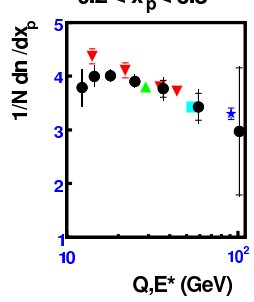

$0.3<x_{p}<0.4$
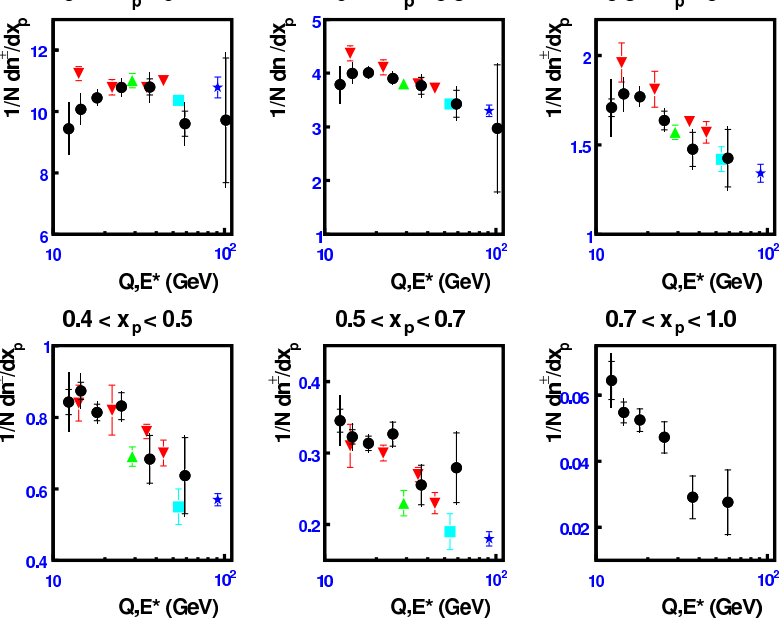

FIG. 1: H1 data for the event-normalised inclusive scaled momentum spectrum, $x_{p}$, as a function of $Q$ for 9 different $x_{p}$ regions. Measurements have been done in the Breit frame of reference for the kinematic region $100<Q^{2}<20000 \mathrm{GeV}^{2}$ and $0.05<y<0.6$. Also shown are data from various $e^{+} e^{-}$experiments (taking $Q=E^{*}-$ the center-of-mass energy).

this case, models require some modifications, for example introducing concepts like underlying events $(p \bar{p})[6]$.

The results on $K_{S}^{0}, \Lambda$ and $\bar{\Lambda}$ production are presented in Fig. 2. This figure shows the strange baryon to meson ratio, $R=(N(\Lambda)+N(\bar{\Lambda})) / N\left(K_{S}^{0}\right)$, where $N(\Lambda), N(\bar{\Lambda}), N\left(K_{S}^{0}\right)$ refer to the number of indicated hadrons. Measurements are based on a data sample about 100 times larger than that used in previous HERA publications [7, 8] and include measurements extenting the phase-space coverage.

The measured and predicted $R$ is shown as a function of $\mathrm{p}_{\mathrm{T}}^{\mathrm{LAB}}$ and $\eta^{\mathrm{LAB}}$ for two DIS samples, and as a function of $\mathrm{p}_{\mathrm{T}}^{\mathrm{LAB}}, \eta^{\mathrm{LAB}}$ and $x_{\gamma}^{\mathrm{OBS}}$ for the photoproduction sample, where 
$x_{\gamma}^{\mathrm{OBS}}$ enrich each component direct and resolved photoproduction. The imperfections in the description of the $K_{S}^{0}$ and $\Lambda+\bar{\Lambda}$ cross-section for high- $Q^{2}$ are clearly reflected in $R$. Overall, however ARIADNE with the strangeness suppression factor of 0.3 follows the shape of the data and is usually in agreement to better than about $10 \%$. The low- $Q^{2}$ sample is described by the same Monte Carlo programs with even a better accuracy. For the direct photoproduction sample, where $x_{\gamma}^{\mathrm{OBS}}>0.75$, the PYTHIA prediction is in agreement with the data but its prediction that $R$ is flat, at about 0.4 , down to smallest $x_{\gamma}^{\mathrm{OBS}}$, is far below the data, which raises steadily to about 0.7 at the smallest $x_{\gamma}^{\mathrm{OBS}}$, corresponding to almost pure resolved photoproduction.

\section{ZEUS}
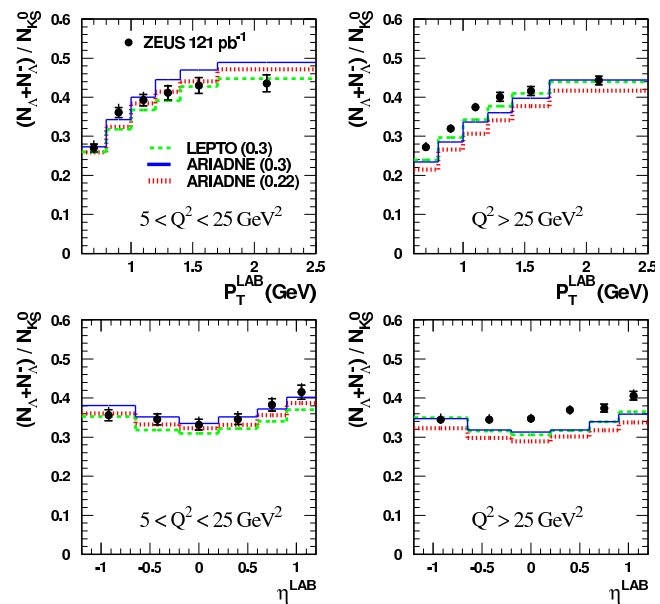

\section{ZEUS}
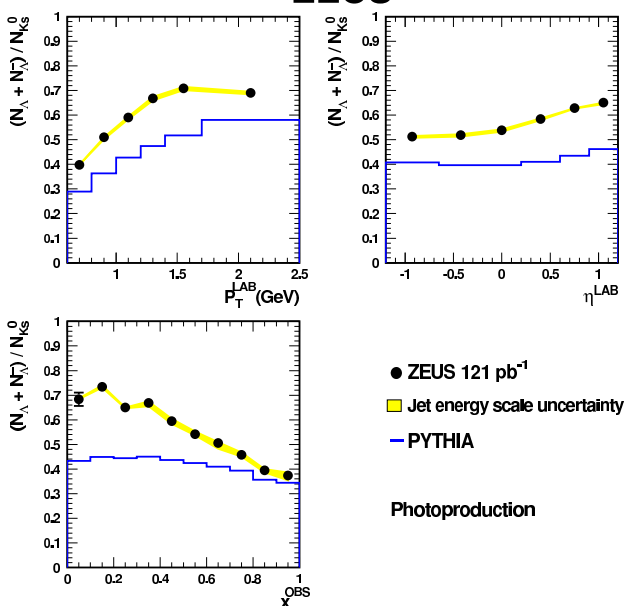

FIG. 2: Strange baryon to meson ratio as a function of $\mathrm{p}_{\mathrm{T}}^{\mathrm{LAB}}$ and $\eta^{\mathrm{LAB}}$ in the range $0.6<\mathrm{p}_{\mathrm{T}}^{\mathrm{LAB}}<2.5 \mathrm{GeV}$ and $\left|\eta^{\mathrm{LAB}}\right|<1.2$ for two DIS samples with $5<Q^{2}<25 \mathrm{GeV}^{2}, 0.02<y<0.95$ and $Q^{2}>$ $25 \mathrm{GeV}^{2}, 0.02<y<0.95$, and as a function of $\mathrm{p}_{\mathrm{T}}^{\mathrm{LAB}}, \eta^{\mathrm{LAB}}$ and $x_{\gamma}^{\mathrm{OBS}}$ ( $x_{\gamma}^{\mathrm{OBS}}$ enrich each component direct and resolved photoproduction) for the photoproduction sample with $Q^{2}<1 \mathrm{GeV}^{2}$ and $0.2<y<$ 0.85 .

\section{BOSE-EINSTEIN CORRELATIONS}

The use of Bose-Einstein correlations (BEC) as the method of determination of the size and the shape of the source from which particles originate has more than forty years history $[9,10]$. BEC originates from symmetrisation of the twoparticle wave function of identical, like-sign bosons and lead to an enhancement of boson pairs emitted with small relative momenta, close to each other in phase-space. This effect is sensitive to the size of the boson emitting source. Such studies have been carried out in a large variety of particle interactions and over a wide range of energies [11-15].
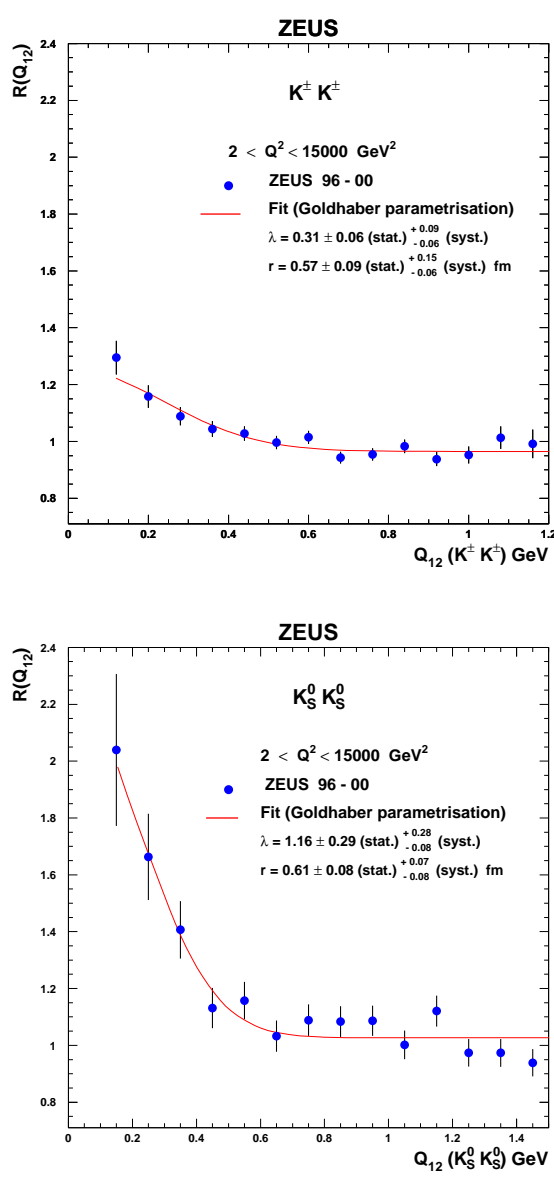

FIG. 3: The two-particle correlation function, $R\left(Q_{12}\right)$, for charged and neutral kaons with fit, $R\left(Q_{12}\right)=\alpha\left(1+\lambda e^{-Q_{12}^{2} r^{2}}\right)\left(1+\beta Q_{12}\right)$.

Figure 3 shows the ratio of the two-particle correlation function of $K^{ \pm} K^{ \pm}$and $K_{S}^{0} K_{S}^{0}$ pairs to the two-particle correlation function of a "reference sample", which is a sample constructed to have no BEC but to retain all other types of correlation. The results obtained by the fit parametrised by the modified Goldhaber function [10],

$$
R\left(Q_{12}\right)=\alpha\left(1+\lambda e^{-Q_{12}^{2} r^{2}}\right)\left(1+\beta Q_{12}\right),
$$

where $r$ is a geometrical radius of the boson emitting source, $\lambda$ is a coherence strength factor, $Q_{12}$ is the four momentum difference of the boson pairs. In this analysis the $\beta$ parameter was found to be zero within errors and was, therefore, ignored. The extracted radius for the BEC correlations for $K^{ \pm} K^{ \pm}$pairs ( $r=0.57 \pm 0.09_{-0.06}^{+0.15} \mathrm{fm}$ ) is consistent with that for charged pions in DIS [16], which is $r=0.666 \pm 0.009_{-0.036}^{+0.022} \mathrm{fm}$. The 
radius for $K_{S}^{0} K_{S}^{0}\left(r=0.61 \pm 0.08_{-0.08}^{+0.07} \mathrm{fm}\right)$ is in good agreement with that for $K^{ \pm} K^{ \pm}$. The radius value for DIS is in good agreement with LEP measurements $[17,18]$.
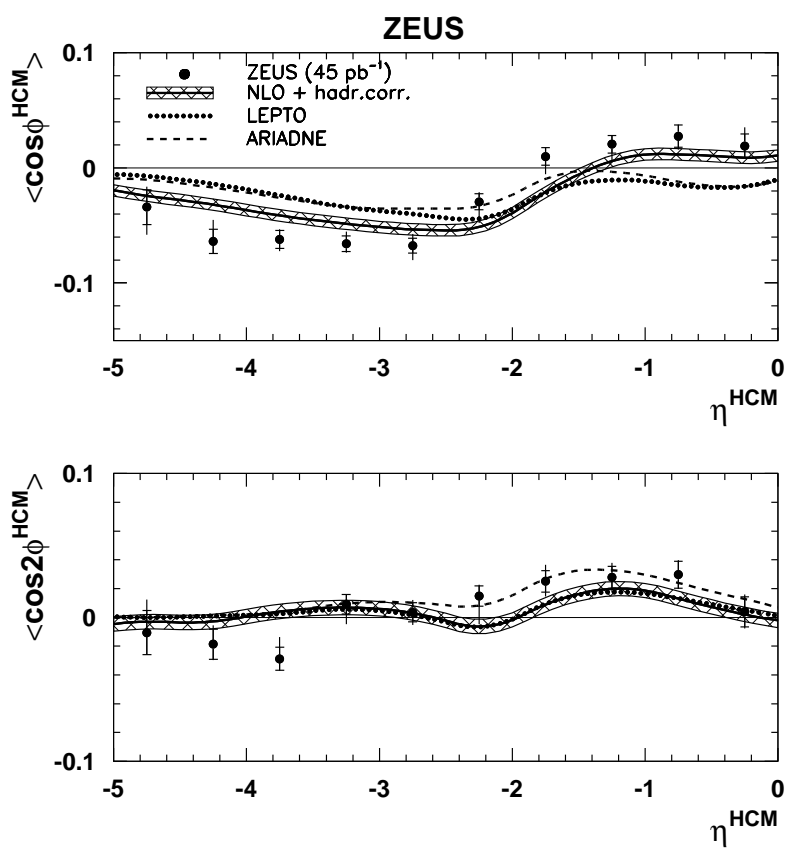

FIG. 4: The values of $\left\langle\cos \phi^{\mathrm{HCM}}\right\rangle$ and $\left\langle\cos 2 \phi^{\mathrm{HCM}}\right\rangle$, calculated using the energy-flow method, as a function of hadron pseudorapidity, $\eta^{\mathrm{HCM}}$. They were obtained in the HCM frame for the kinematic region $100<Q^{2}<8000 \mathrm{GeV}^{2}, 0.01<x<0.1$ and $0.2<y<0.8$.

\section{AZIMUTHAL ASYMMETRY}

The azimuthal angle, $\phi$, is defined in the hadronic center-ofmass frame, HCM, as the angle between the lepton scattering plane, defined by the incoming and outgoing lepton momenta, and the hadron production plane, defined by the exchanged virtual photon and an outgoing hadron. In pQCD, higher- order QCD processes such as QCD Compton (QCDC) and boson-gluon fusion (BGF) are the main sources of asymmetries in $\phi$. These two processes have different $\phi$ behaviors [19] as well as a different pseudorapidity, $\eta$, dependence, defined here with respect to the incoming proton direction in the HCM frame. The azimuthal dependence for semi-inclusive neutral current (NC) DIS has the form [20-23],

$$
d \sigma / d \phi=A+B \cos \phi+C \cos 2 \phi+D \sin \phi+E \sin 2 \phi .
$$

This form results from the polarization of the exchanged virtual photon. The coefficient $B$ originates from the interference between the transversely and longitudinally polarized components; the coefficient $C$ is due to the interference of amplitudes corresponding to the +1 and -1 helicity parts of the transversely polarized photon. The coefficients $D$ and $E$ arise from parity-violating weak interactions or longitudinal polarization of the initial lepton beam [21]. They vanish for purely electromagnetic interactions with unpolarized beams.

The mean values of $\cos \phi^{\mathrm{HCM}}$ and $\cos 2 \phi^{\mathrm{HCM}}$, are shown in Fig. 4 as a function of $\eta^{\mathrm{HCM}}$. An energy-flow analysis method was used, which permitted the use of both neutral and charged hadrons and extends the phase space over previous measurements $[24,25]$. The value of $\left\langle\cos \phi^{\mathrm{HCM}}\right\rangle$ is negative for $\eta^{\mathrm{HCM}}<-2$ but becomes positive for larger $\eta^{\mathrm{HCM}}$. This is in disagreement with the ARIADNE and LEPTO predictions. The data agree better with the next-to-leading-order (NLO) predictions, corrected for hadronization. The measured $\left\langle\cos 2 \phi^{\mathrm{HCM}}\right\rangle$ values are consistent with zero for $\eta^{\mathrm{HCM}}<$ -2 but are positive for higher values of $\eta^{\mathrm{HCM}}$. This is described by ARIADNE and LEPTO. The values of $\left\langle\sin \phi^{\mathrm{HCM}}\right\rangle$ and $\left\langle\sin 2 \phi^{\mathrm{HCM}}\right\rangle$ are consistent with zero (not shown).

\section{Acknowledgements}

I thank the DESY Directorate for their strong support and encouragement. Special thanks go to Teresa Tymieniecka, Yuji Yamazaki and my wife Justyna for invaluable help.
[1] EMC, M. Arneodo et al., Z. Phys. C 35, 417 (1987).

[2] H1 Coll., C. Adloff et al., Nucl. Phys. B 504, 3 (1997).

[3] H1 Coll., C. Adloff et al., in ICHEP'98: Proceedings, ed. A. Astbury et al., World Scientific (Singapore 1999), p. 531.

[4] ZEUS Coll., M. Derrick et al., Phys. Lett. B 414, 428 (1997).

[5] ZEUS Coll., J. Breitweg et al., Eur. Phys. J. C 11, 251 (1999).

[6] CDF Coll., D. Acosta et al., Phys. Rev. D 71, 112002 (2002).

[7] H1 Coll., C. Adloff et al., Z. Phys. C 76, 213 (1997).

[8] ZEUS Coll., J. Breitweg et al., Eur. Phys. J. C 2, 77 (1998).

[9] G. Goldhaber et al., Phys. Rev. Lett. 3, 181 (1959).

[10] G. Goldhaber et al., Phys. Rev. 120, 300 (1960).

[11] G. Alexander, Acta Phys. Pol. B 35, 69 (2004).

[12] G. Alexander, Rep. Prog. Phys. 66, 481 (2003).

[13] T. Csörgo, Heavy Ion Phys. 15, 1 (2002).

[14] T. Csörgo, J. Phys. Conf. Ser. 50, 259 (2006).

[15] E-802 Coll., T. Abbott et al., Phys. Rev. Lett. 69, 1030 (1992).
[16] ZEUS Coll., S. Chekanov et al., Phys. Lett. B 583, 231 (2004).

[17] DELPHI Coll., S. Abreu et al., Phys. Lett B 379, 330 (1996).

[18] OPAL Coll., G. Abbiendi et al., Eur. Phys. J. C 21, 23 (2001).

[19] V. Hedberg et al., Proc. Workshop on Physics at HERA, W. Buchmüller and G. Ingelman (eds.), Vol. 1, p. 331, Hamburg, Germany, DESY (1992).

[20] A. Méndez, Nucl. Phys. B 145, 199 (1978).

[21] K. Hagiwara, K.I. Hikasa, and N. Kai, Phys. Rev. D 27, 84 (1983).

[22] J. Chay, S.D. Ellis, and W.J. Stirling, Phys. Lett. B 269, 175 (1991).

[23] M. Ahmed and T. Gehrmann, Phys. Lett. B 465, 297 (1999).

[24] ZEUS Coll., J. Breitweg et al., Phys. Lett. B 481, 199 (2000).

[25] ZEUS Coll., S. Chekanov et al., Phys. Lett. B 551, 3 (2003). 